\title{
FEM Analysis of Ocean Current Effects on the Postures of Submerged Buoys
}

\author{
Wang Zhongqiu ${ }^{1}$, Wang Yiming $^{2}$, Wang Hongru ${ }^{1}$, Zheng $\mathrm{Yi}^{1}$ \\ 1. Shandong Provincial Key Laboratory of Ocean Environment Monitoring Technology, Shandong Academy of Sciences \\ Institute of Oceanographic Instrumentation, Qingdao, China, 266001 \\ 2. The First Institute of Oceanography SOA, Qingdao, China, 266001
}

\begin{abstract}
The ocean current affection on the posture of two type of submerged buoy is analyzed through FEM analysis. By comparing the factors of impact pressure coefficient distribution, ocean current speed distribution, impact pressure force in horizontal direction and vertical direction, Turbulence Kinetic Energy distribution and vortex generation, the advantage and disadvantage of the two structure are compared. By considering these affect factors, the horizontal buoy structure is better than horizontal buoy structure.
\end{abstract} analysis

Keywords-FEM,Submerged buoy,Ocean current,Posture

\section{INTRODUCTION}

Submerged buoy is a widely used instrument in many marine investigation conditions. The posture of buoy under various ocean current lashing is a very important factor that can affect the function of the instruments mounted on it. Besides, ocean current impacting on the buoy body will generate turbulence and vortex; so many researchers have done many works in this field, such as submarine hydrodynamics analysis [1-3]. As the development of CFD technology, the fluid field around the target under ocean current could be calculated numerically instead of water tunnel experiment or on-site test in the ocean $[4,5]$. In this paper, the ocean current on the posture of submerged buoy is analyzed using FEM method, to verify the advantage and disadvantage of the two structures.

\section{CALCUlation THEORY AND MATHIMATIC MODEL}

For the $3 \mathrm{D}$ incompressible viscous fluid, the dimensionless tensor form of RANS equation under the Cartesian coordinates system could be described as follows:

Continuous equation:

$$
\frac{\partial u_{i}}{\partial x_{i}}=0
$$

Momentum equation:

$$
\frac{\partial u_{i}}{\partial t}+u_{j} \frac{\partial u_{i}}{\partial x_{j}}=F_{i}-\frac{1}{\rho} \frac{\partial p}{\partial x_{j}}+\frac{\partial}{\partial x_{j}}\left(v \frac{\partial u_{i}}{\partial x_{j}}-\overline{u_{i}^{\prime} u_{j}^{\prime}}\right)
$$

Where, $\rho$ is the density of fluid, $v$ is the kinematic viscosity coefficient of fluid, $F_{i}$ is outer force term, $u_{i}$ is average velocity, $p$ is average pressure, ${ }^{\prime}{ }_{i}^{\prime}$ is the velocity pulsation value., in order to take the average of the N-S equation by time, the unknown Reynolds stress term $\overline{u_{i}^{\prime} u_{j}^{\prime}}$ is introduced and the turbulence model is needed helping to enclose the equation.

$$
\begin{aligned}
& -\overline{u_{i}^{\prime} u_{j}^{\prime}}=v_{t}\left(\frac{\partial u_{i}}{\partial x_{j}}+\frac{\partial u_{j}}{\partial x_{i}}\right)-\frac{2}{3} k \delta_{i j} \\
& v_{t}=C_{\mu} \frac{K^{2}}{\varepsilon}
\end{aligned}
$$

Where $\delta_{i j}$ is Kronecker symbol, $v_{t}$ is turbulent viscosity, $C_{\mu}$ is constant, $K$ is turbulent energy and $\varepsilon$ is turbulent diffusion coefficient. In this paper, the twoequation turbulent $R N G k-\varepsilon$ model is used. The turbulent energy equation and turbulent diffusion coefficient equation are described as equation(5), (6):

$$
\begin{aligned}
& \frac{\partial}{\partial_{t}}(\rho k)+\frac{\partial}{\partial x_{i}}\left(\rho k u_{i}\right)=\frac{\partial}{\partial x_{j}}\left[\alpha_{k} \mu_{e f f} \frac{\partial k}{\partial x_{J}}\right]+G_{k}+G_{b}-\rho \varepsilon-Y_{M}+S_{k} \\
& \frac{\partial}{\partial_{t}}(\rho \varepsilon)+\frac{\partial}{\partial x_{i}}\left(\rho \varepsilon u_{i}\right)= \\
& \frac{\partial}{\partial x_{j}}\left[\alpha_{k} \mu_{e f f} \frac{\partial \varepsilon}{\partial x_{J}}\right]+C_{1 \varepsilon} \frac{\varepsilon}{k}\left(G_{k}+G_{3 \varepsilon} G_{b}\right)-C_{2 \varepsilon} \rho \frac{\varepsilon^{2}}{K}-R_{\varepsilon}+S_{\varepsilon}
\end{aligned}
$$

Where $G_{k}$ is the turbulent energy generated by laminar flow velocity gradient, $G_{b}$ is the turbulent energy generated by buoyancy, $Y_{m}$ is the compressible turbulent vibration generating during transition. Constant value $C_{1 \varepsilon}=1.42$, $C_{2 \varepsilon}=1.68$, the parameter $\alpha_{k}$ and ${ }^{\alpha_{\varepsilon}}$ are the turbulent Prandtt number of equation, $S_{k}$ and $S_{\varepsilon}$ is define by user, in this paper the two value are 0 .

\section{RESEARCH CONTENTS}

In order to investigate the influence of ocean current on the postures of submerged buoy, two types of buoy are designed, which are vertical buoy and longitudinal buoy, as is shown in Figure 1. The basic dimensions for the two structures are listed in

. Since the submerged buoy will be enclosed by fairwater cone to prevent the water directly impact on the sensors mounted in the buoy, the two models will be simplified to outer envelope surface of fairwater cone. 


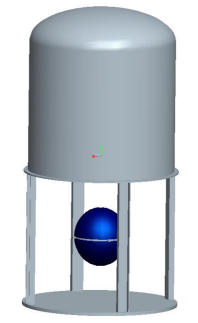

a) vertical style buoy

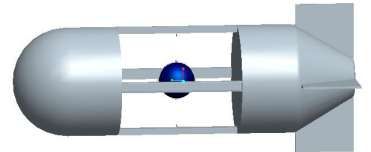

b) longitudinal style buoy
Figure 1. two types of buoy structure

TABLE I. CUTTING PARAMETERS OF SIMULATION MILLING

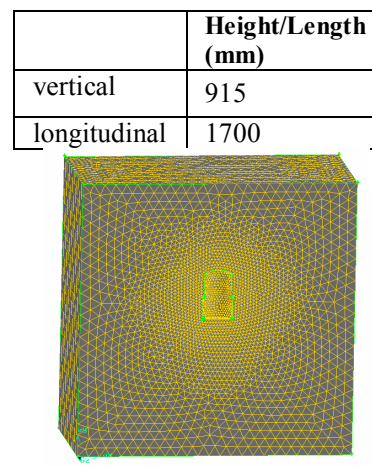

a) mesh of vertical style buoy

\begin{tabular}{|c|c|}
\hline $\begin{array}{l}\begin{array}{l}\text { Diameter } \\
(\mathrm{mm})\end{array} \\
\end{array}$ & $\begin{array}{l}\text { Ocean current } \\
(\mathrm{m} / \mathrm{s})\end{array}$ \\
\hline 500 & \multirow{2}{*}{$0.5,1,2,3,4$} \\
\hline 533 & \\
\hline
\end{tabular}

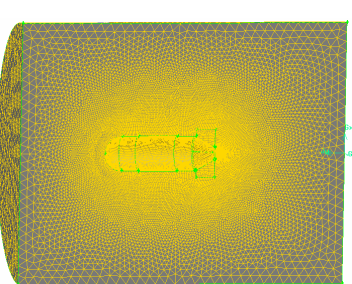

b) mesh of longitudinal style buoy
Figure 2. mesh conditions of two types of buoy

In simulation, only 5 times more volume space area compared with buoy is enough. Ocean current comes from the left side of the area, and flows out from the right side, the simulation process neglect the gravity. The meshing results are shown in Figure 2, the buoys are meshed with the element size of 0.02 relative size to gain enough calculation precision and the outer area are meshed with auto scale meshing with the maximum mesh size of 0.15 to save calculation time.

\section{RESUlTS AND DisCUSSION}

It can be seen from the comparison in Figure 3 that, for the vertical type buoy, the front side and back side of buoy suffer larger pressure coefficient than the middle area and the bottom side also suffers larger pressure coefficient than the top side as is shown in Figure 3-a). This phenomenon is reasonable, in the fluid flow direction, since the flow at the front and back side of buoy are small because of the block of the body, but at the two sides in the middle, the fluid can flow over around the arc of the body; in the vertical direction, since the envelope on the top is streamlined, fluid flow resistance is smaller than the straight shaped bottom, so the fluid speed in the top side is larger than the bottom side as is shown in Figure 4-a). For the horizontal type buoy as is shown in Figure 3-b)., the trend of pressure coefficient variation along the flow direction is the same with the vertical type, but since the horizontal type buoy is symmetry in the vertical direction, so there is no difference in the top and bottom side as is shown in Figure 4-b).

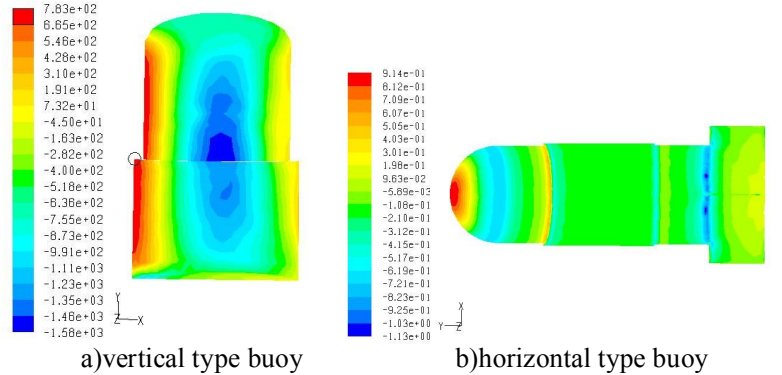

Figure 3. pressure coefficient comparison with ocean current speed $\mathrm{v}=1 \mathrm{~m} / \mathrm{s}$
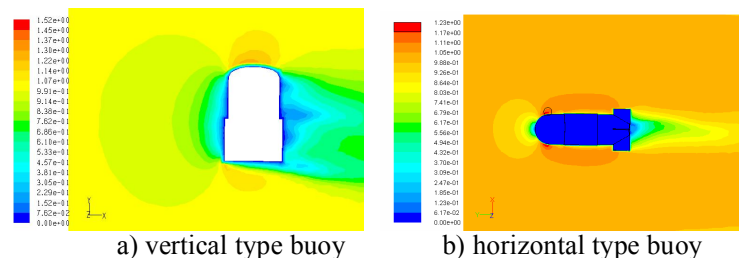

b) horizontal type buoy

Figure 4. velocity comparison with ocean current speed $\mathrm{v}=1 \mathrm{~m} / \mathrm{s}$

Figure 5 is the ocean current force applied on the buoys in horizontal and vertical direction at different sea fluid speed. It can be seen from the figure that, the impact force in the horizontal direction increases nonlinear as the ocean current speed increases for both type of buoy, but impact force in the vertical direction is opposite. The impact force applied on the vertical type buoy is upward and this will add extra buoyancy force on the buoy causing the mooring cable tight. On the contrary, the horizontal type buoy suffers downwards impact force in vertical direction, but the absolute impact force value is very small, this opposite force will not affect buoyancy force much. Since the vertical type buoy suffers much larger impact force than horizontal type buoy, the magnitude order is almost 2500 times. In some real case, the absolute buoyancy force is about $1500 \mathrm{~N}$ and the tilt angle should not larger than $1.5^{\circ}$. The impact force in the horizontal direction will change the buoy posture as the ocean current speed increases, as is shown in Figure 6. For this case, the horizontal type buoy will not applicable when the ocean current speed is larger than $1 \mathrm{~m} / \mathrm{s}$, which is very critical. For the horizontal type buoy, the impact force will not affect the posture much in any direction, and the impact force could be neglected.
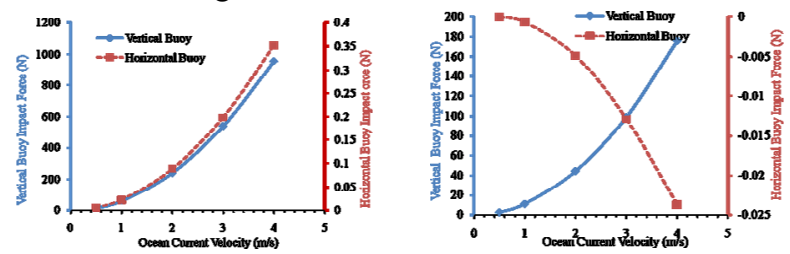

a) impact force in horizontal direction b) impact force in vertical direction

Figure 5. impact force comparison of two types of buoy unssder different ocean current speed 


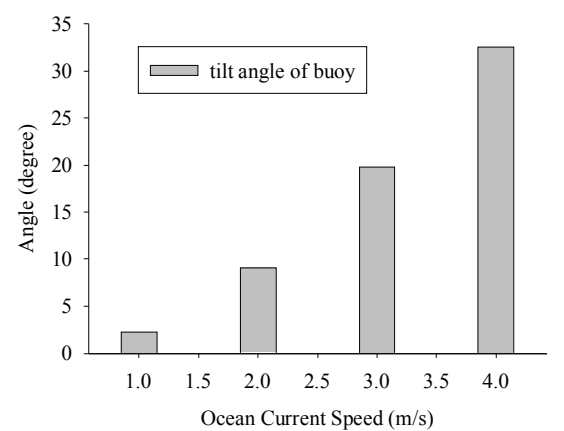

Figure 6. tile angle of vertical type buoy under different ocean current speed

Figure 7 is the comparison of Turbulence Kinetic Energy of these two types of buoy at the ocean current speed of $2 \mathrm{~m} / \mathrm{s}$. From the figure it can be seen that, the maximum Turbulence Kinetic Energy of vertical buoy is smaller than horizontal buoy, but the distribution area of vertical buoy is much larger than horizontal buoy, the Turbulence Kinetic Energy distributed close to the surface of horizontal buoy. Though the maximum value of horizontal buoy is a little larger than that of vertical buoy, the distribution layer is very small, so that the current Turbulence Kinetic Energy along the surface will dissipate quickly without vortex generation near the front surface but only one vortex generated at the back of tail. For the vertical buoy, the Turbulence Kinetic Energy distributed very wide at the back of buoy, so it is possible to generate vortex at the center of turbulence center, which is verified by path line analysis that is not shown. There are three vortexes generate at the back of vertical buoy at the maximum value area in Figure 7-a), but only one vortex at the tail back of horizontal buoy.

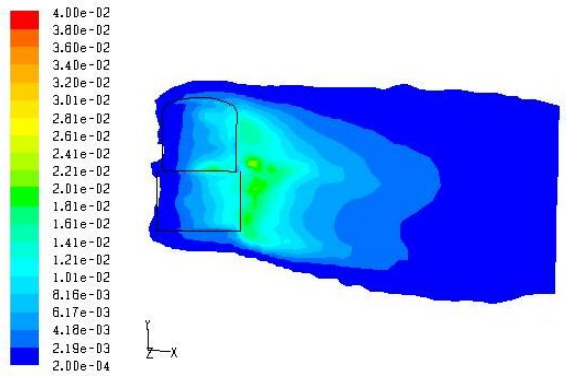

a) vertical type buoy

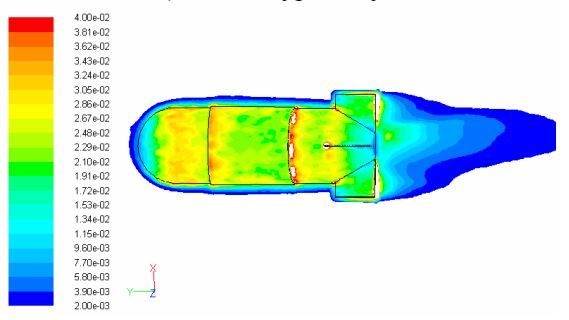

b) horizontal type buoy

Figure 7. comparison of Turbulence Kinetic Energy K with ocean current speed $\mathrm{v}=2 \mathrm{~m} / \mathrm{s}$

\section{SUMmaries AND CONCLUSIONS}

From the analysis above, it can be concluded that:

(1) The vertical buoy and horizontal buoy has the same pressure distribution trend along the ocean current direction since, but the pressure of vertical buoy at vertical direction is different since the unsymmetrical structure.

(2) The vertical buoy suffers large impact forces under ocean current than horizontal buoy especially in ocean current direction; this will change the tilt angle of buoy. When the facilities mounted on the buoy have critical constrain of tilt angle, the vertical structure will not applicable. However, the horizontal structure buoy suffers nearly negligible impact forces in both horizontal (ocean current direction) and vertical direction. This structure is stable in the ocean current direction, but since this structure is longitudinal, which will change the direction as the ocean current direction changes. If there is facilities with direction determination function, extra compass is needed for correction.

(3) Regarding Turbulence Energy of ocean current, the vertical buoy has smaller Turbulence Energy than horizontal buoy, but the distribution area is much large, especially at the middle back of vertical buoy, there are more vortexes generated for vertical buoy than that of horizontal buoy, which may lead to more hydrodynamic noise and posture vibration. But the horizontal buoy only has one vortex at the back of tail, and the affecting area is very limited.

So considering the ocean current affections on the posture of buoy, horizontal structure buoy is better choice design than vertical buoy. This structure doesn't need extra design for the posture statistics.

\section{ACKNOWLEDGMENT}

This paper was supported by the National Natural Science Foundation of China under Grant 40806044, Supported by the Qingdao Science and Technology Planning Project Fund, China under Grant 10-3-4-9-2-jch and Supported by Research Fund for the Doctoral Program of the Shandong Academy of Sciences under Grant Y09-2.

\section{REFERENCES}

[1] Atkins D.J. Computational hydrodynamics for submarine-recent development. International Symposium on Naval Submarine 5 [C], 1996.10

[2] Prince W.G. Fluid forces on maneuvering submarines and bodyappendage configurations. Warship'93 ISNS[C], 1993,pp:1-14.

[3] Levy R. Analysis of the submarine appendage flow field. ADA212344[R], 1998. 21.

[4] P. Bull. The Validation of CFD Predictions of Nominal Wake for the SUBOFF Fully Appended Geometry. Twenty-First Symposium on Naval Hydrodynamics, 1997, PP: 1061-1076.

[5] ZHANG Nan, SHEN Hong-cui, YAO Hui-zhi, Ma Li-jun. Numerical simulation of flow around submarine and the scaling effect of high Reynolds number on flow field. CSHydro 2007 Conference [C], PP: 738-748. 